\title{
Structural Similarity Measure to Assess Improvement by Noise in Nonlinear Image Transmission
}

\author{
David Rousseau, Agnès Delahaies, and François Chapeau-Blondeau
}

\begin{abstract}
We show that the structural similarity index is able to register stochastic resonance or improvement by noise in nonlinear image transmission, and sometimes when not registered by traditional measures of image similarity, and that in this task this index remains in good match with the visual appreciation of image quality.
\end{abstract}

Index Terms-Image quality measure, improvement by noise, nonlinearity, stochastic resonance, structural similarity.

\section{INTRODUCTION}

A UTOMATIC image quality assessment is an important task for many areas of image processing. Traditional metrics commonly used for image quality, such as mean squared error, peak signal-to-noise ratio and related indices, are simple to implement but are limited to providing a low-level-based assessment of images. Recently, the structural similarity (SSIM) index has been proposed as a novel measure of image quality [1], [2], with the potentiality for a more structural assessment based on evaluating image degradation as a combination of three different factors: loss of correlation, luminance and contrast distortions. The SSIM index has been shown to outperform simpler traditional metrics while avoiding the complexity of an explicit modeling of perception by the human visual system [1], [2]. This ability of SSIM to offer an efficient measure of image quality was established in [1], [2] in various image processing tasks such as compression, contrast stretching, mean shifting, noise contamination, blurring, and it was tested against visual appreciation of image quality. Yet, as a recently introduced index, SSIM may still benefit, as advocated in [1], from further experiments in order to fully appreciate its capabilities. In the present paper, we test SSIM as a relevant index to measure stochastic resonance or improvement by noise in nonlinear image transmission.

Stochastic resonance, in broad sense, designates situations where the noise can play a constructive role in signal processing [3]-[5]. Such possibility to occur usually demands the presence of a nonlinear process. Stochastic resonance or improvement by noise has been shown possible in different types of nonlinear image transmission or processing [6]-[11]. The most common indices that were used to manifest stochastic resonance

Manuscript received June 18, 2009; revised August 15, 2009. First published September 09, 2009; current version published October 14, 2009. The associate editor coordinating the review of this manuscript and approving it for publication was Dr. Alfred Mertins.

The authors are with the Laboratoire d'Ingénierie des Systèmes Automatisés (LISA), Université d'Angers, 49000 Angers, France (e-mail: chapeau@univangers.fr).

Digital Object Identifier 10.1109/LSP.2009.2031734 for aperiodic signals or images were traditional similarity indices like correlation measures or mean squared error. Such an index measures the similarity between an input image and an output image resulting from nonlinear transmission in the presence of noise, and stochastic resonance is manifested by the input-output similarity index which culminates at a maximum occurring for a finite nonzero amount of noise. This identifies an improvement by noise of a nonlinear image transmission, yet with low-level-based indices of image quality. At the same time, stochastic resonance has been registered in psychovisual experiments when measuring the performance by visual perception of human subjects [6], [12]-[14]. Here, we complement the measures of stochastic resonance by testing the SSIM index of [1], [2] for the first time for this purpose.

Also, most reports on stochastic resonance with images have dealt with threshold or potential-barrier nonlinearities, where the noise essentially assists a small signal in overcoming a threshold or barrier. In this context of threshold nonlinearities, more specific techniques like dithering can be viewed as a special form of stochastic resonance [15]-[17]. Yet, stochastic resonance can occur in other types of nonlinearities, and we show it here with saturation as well as threshold nonlinearities. Saturation nonlinearities are common in imaging devices, and while testing the SSIM index here, we also illustrate the possibility of image saturation restored by noise.

\section{Similarity MEASURES}

For two images $\mathbf{x}=\left\{x_{i} \mid i=1,2, \ldots N\right\}$ and $\mathbf{y}=\left\{y_{i} \mid i=\right.$ $1,2, \ldots N\}$ with same size, we consider the SSIM index $S(\mathbf{x}, \mathbf{y})$ defined from [1] as

$$
S(\mathbf{x}, \mathbf{y})=\frac{4(\langle x y\rangle-\langle x\rangle\langle y\rangle)\langle x\rangle\langle y\rangle}{\left(\left\langle x^{2}\right\rangle-\langle x\rangle^{2}+\left\langle y^{2}\right\rangle-\langle y\rangle^{2}\right)\left(\langle x\rangle^{2}+\langle y\rangle^{2}\right)}
$$

where $\langle\cdot\rangle$ stands for an average over the images, for instance $\langle x y\rangle=N^{-1} \sum_{i=1}^{N} x_{i} y_{i}$. The SSIM index $S(\mathbf{x}, \mathbf{y})$ of (1) varies in $[-1,1]$; it achieves its best value of 1 when the two images $\mathbf{X}$ and $\mathbf{y}$ are equal; it is at zero when $\mathbf{x}$ and $\mathbf{y}$ are unrelated; it is at -1 when $\mathbf{x}$ and $\mathbf{y}$ are equal except for opposite signs of their fluctuations. References [1], [2], [18] motivate the definition of $S(\mathbf{x}, \mathbf{y})$ to quantify the structural departure between images $\mathbf{x}$ and $\mathbf{y}$ by combining loss of correlation, luminance and contrast distortions. In addition, we consider the two traditional measures given by the cross-correlation coefficient

$$
C(\mathbf{x}, \mathbf{y})=\frac{\langle x y\rangle-\langle x\rangle\langle y\rangle}{\sqrt{\left\langle x^{2}\right\rangle-\langle x\rangle^{2}} \sqrt{\left\langle y^{2}\right\rangle-\langle y\rangle^{2}}}
$$

and root mean squared (rms) error 


$$
E(\mathbf{x}, \mathbf{y})=\sqrt{\left\langle(x-y)^{2}\right\rangle} .
$$

Additionally, $S(\mathbf{x}, \mathbf{y})$ can be factored as $S=\mathrm{CMV}$ where $M(\mathbf{x}, \mathbf{y})=2\langle x\rangle\langle y\rangle /\left(\langle x\rangle^{2}+\langle y\rangle^{2}\right)$ measures the similarity in the means of $\mathbf{x}$ and $\mathbf{y}$, and $V(\mathbf{x}, \mathbf{y})=$ $2 \sqrt{\left\langle x^{2}\right\rangle-\langle x\rangle^{2}} \sqrt{\left\langle y^{2}\right\rangle-\langle y\rangle^{2}} /\left(\left\langle x^{2}\right\rangle-\langle x\rangle^{2}+\left\langle y^{2}\right\rangle-\langle y\rangle^{2}\right)$ measures the similarity in their contrasts [1].

We use and confront the three indices $S, C$ and $E$ of (1)-(3) to assess image transmission by nonlinear devices in the presence of noise. We specifically study the regime, related to a stochastic resonance effect, where the noise can play a constructive role in the nonlinear transmission. An input image $\mathbf{x}$ is corrupted by an additive noise $\mathbf{n}$ with the noise realizations assumed independent at each pixel. The noisy image $\mathbf{x}+\mathbf{n}$ is then acquired or transmitted by a nonlinear device modeled by the memoryless characteristic $g(\cdot)$ which delivers the output image $\mathbf{y}$ defined as

$$
\mathbf{y}=g(\mathbf{x}+\mathbf{n})
$$

\section{HARD-LIMITER TRANSMISSION}

As a first example, we consider $g(\cdot)$ a hard limiter with threshold $\theta$,

$$
g(u)= \begin{cases}0, & \text { for } u \leq \theta \\ 1, & \text { for } u>\theta\end{cases}
$$

in charge of the transmission of binary images $\mathbf{x}$. We consider the case of a small-amplitude input image $\mathbf{x}$ with binary intensities at 0 or 1 , which is everywhere below the threshold $\theta>1$ of the transmission device of (5). In this condition, in absence of the noise $\mathbf{n}$ in (4), no transmission occurs and at the output $\mathbf{y}$ remains a blank image. Addition of the noise $\mathbf{n}$ in (4) enables a cooperative effect where the noise assists the subthreshold input image $\mathbf{x}$ in overcoming the threshold $\theta$, so as to elicit an output image $\mathbf{y}$ carrying some similarity with the input image $\mathbf{x}$. This similarity is quantified in Fig. 1 with the three measures of (1)-(3), and as a function of the level of the noise $\mathbf{n}$.

In Fig. 1, each similarity index $S, C$ or $E$ experiences a nonmonotonic evolution as the noise level increases, passing through an extremum which identifies a nonzero optimal noise level where the similarity index is at its best. The optimal noise level is different for each index, expressing the notion that each index conveys some distinct aspect concerning the similarity. As yet another aspect, Fig. 2 provides a visual appreciation of the similarity at different levels of noise.

The sequence of images in Fig. 2 also makes clearly visible a nonmonotonic action of the noise $\mathbf{n}$, with a visually poor image transmission at low or high noise levels, and better quality of transmission in between. From Fig. 2, it can be argued that the SSIM index $S(\mathbf{x}, \mathbf{y})$ and the cross-correlation $C(\mathbf{x}, \mathbf{y})$ best match the visual assessment of the images, since images with good visual quality are obtained at the noise levels that maximize $S(\mathbf{x}, \mathbf{y})$ and $C(\mathbf{x}, \mathbf{y})$. By contrast, at the noise level minimizing the rms error $E(\mathbf{x}, \mathbf{y})$, the visual quality appears poorer in Fig. 2, expressing that the rms error is not here in good match with the visual appreciation.

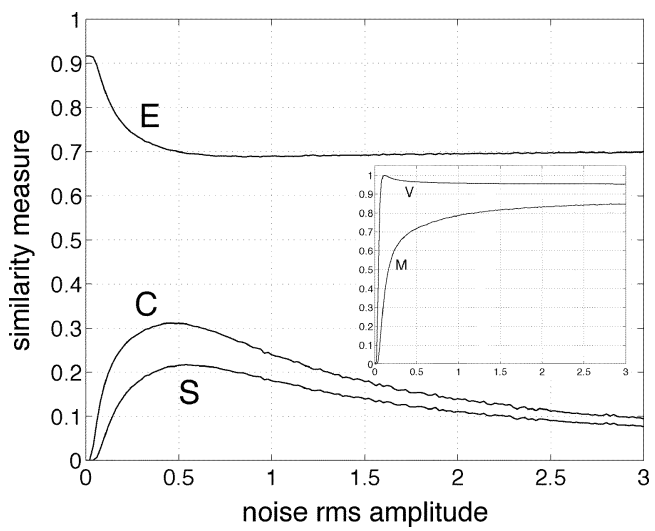

Fig. 1. For the transmission by (5) with threshold $\theta=1.1$ of the binary image $\mathbf{x}$ of Fig. 2(a): as a function of the rms amplitude $\sigma$ of the zero-mean Gaussian noise $\mathbf{n}$ in (4), the input-output similarity indices $S(\mathbf{x}, \mathbf{y})$ of (1), $C(\mathbf{x}, \mathbf{y})$ of (2) and $E(\mathbf{x}, \mathbf{y})$ of (3). The inset shows the partial measures $M(\mathbf{x}, \mathbf{y})$ and $V(\mathbf{x}, \mathbf{y})$ versus $\sigma$.

\section{TRANSMISSION With SATURATION}

We now consider $g(\cdot)$ as a sensor which remains linear for small positive intensities but saturates when the intensities exceed some level $\theta$, i.e.,

$$
g(u)= \begin{cases}0, & \text { for } u<0 \\ u, & \text { for } 0 \leq u \leq \theta \\ \theta, & \text { for } u>\theta\end{cases}
$$

in charge of the transmission of gray-level images $\mathbf{x}$. We consider the case of a high-amplitude input image $\mathbf{x}$ with intensities in $[0,1]$, and which strongly saturates the sensor of (6) having a saturation level $\theta<1$. The resulting output image $\mathbf{y}$ is strongly affected by saturation. The impact of the added noise $\mathbf{n}$ in (4) is shown in Fig. 3 on the three input-output similarity indices of (1)-(3).

In Fig. 3 only the SSIM index $S(\mathbf{x}, \mathbf{y})$ achieves its best value at a nonzero level of the noise $\mathbf{n}$. This expresses the possibility of a constructive action of the noise to improve image transmission in the presence of strong saturation of the sensor, as assessed by SSIM. On the contrary in Fig. 3, the cross-correlation $C(\mathbf{x}, \mathbf{y})$ and the rms error $E(\mathbf{x}, \mathbf{y})$ are at their best with no added noise $\mathbf{n}$, and in this respect do not manifest the possibility of a constructive action of noise in the nonlinear transmission. This again illustrates the notion that each index reflects some distinct aspect concerning the similarity.

Next, a visual appreciation regarding the impact of noise on the input-output similarity can be obtained from Fig. 4.

Again, in Fig. 4 the visual appreciation appears in good match with the behavior of the SSIM index $S(\mathbf{x}, \mathbf{y})$, while the crosscorrelation $C(\mathbf{x}, \mathbf{y})$ and rms error $E(\mathbf{x}, \mathbf{y})$ are comparatively less in tune with the visual appreciation. This is so because, with no added noise, $C(\mathbf{x}, \mathbf{y})$ and $E(\mathbf{x}, \mathbf{y})$ are at their best as shown in Fig. 4, yet from visual inspection in Fig. 4(b) significant features are missing in the output image $\mathbf{y}$ due to saturation. By contrast, missing features become perceivable in Fig. 4 as noise is added manifesting its ability to counteract the negative effect of the saturation. This restoration by noise visually appears specially efficient in Fig. 4(d) at the nonzero noise level maximizing the SSIM index $S(\mathbf{x}, \mathbf{y})$ in Fig. 3. 


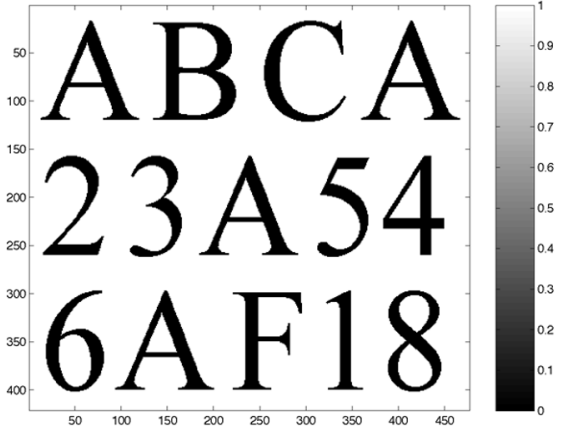

(a)

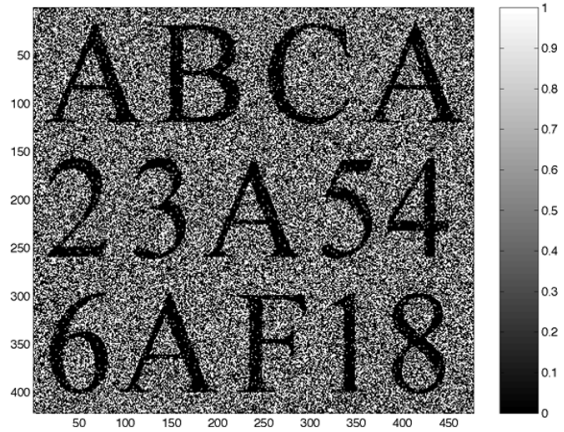

(d)

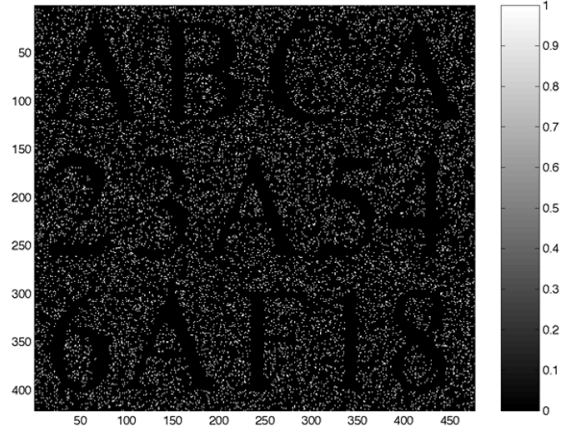

(b)

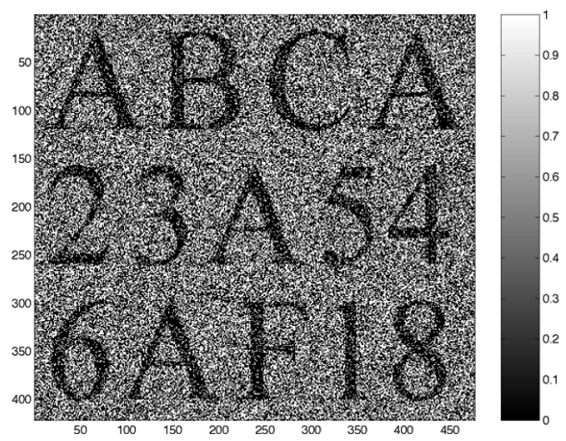

(e)

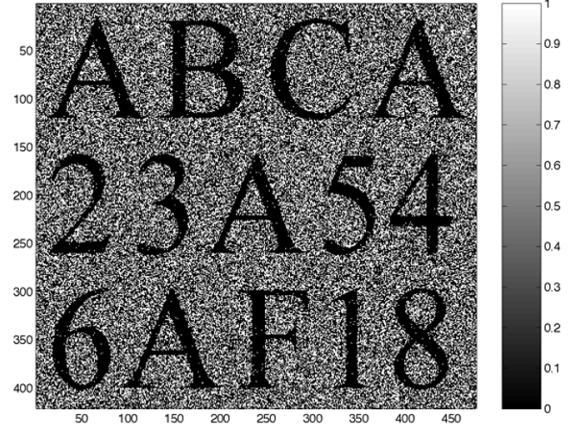

(c)

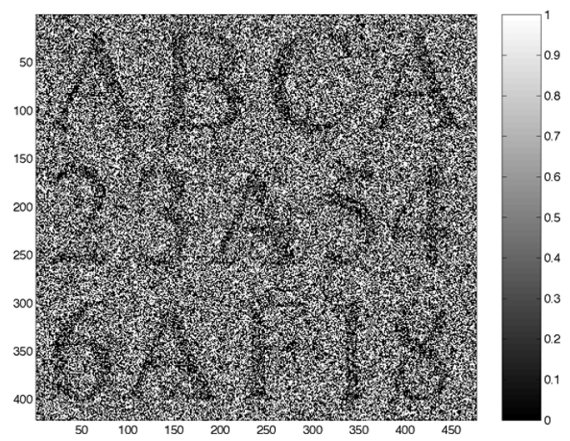

(f)

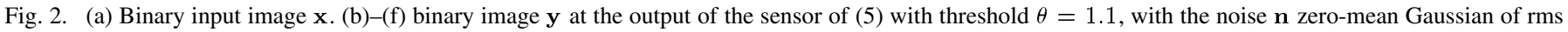

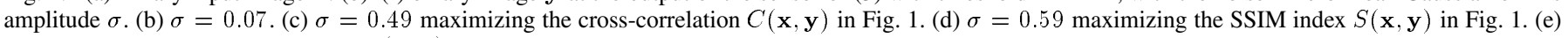
$\sigma=0.99$ minimizing the rms error $E(\mathbf{x}, \mathbf{y})$ in Fig. 1. (f) $\sigma=1.5$.

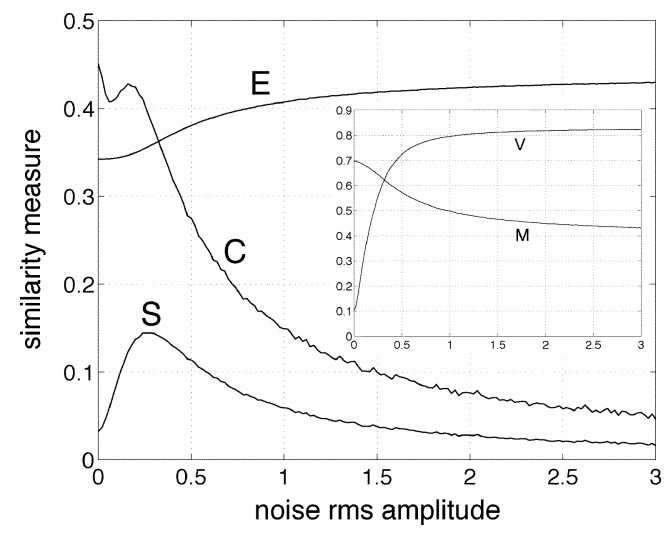

Fig. 3. For the transmission by (6) with saturation level $\theta=0.2$ of the graylevel image $\mathbf{x}$ of Fig. 4(a): as a function of the rms amplitude $\sigma$ of the zeromean Gaussian noise $\mathbf{n}$ in (4), the input-output similarity indices $S(\mathbf{x}, \mathbf{y})$ of (1), $C(\mathbf{x}, \mathbf{y})$ of (2) and $E(\mathbf{x}, \mathbf{y})$ of (3). The inset shows the partial measures $M(\mathbf{x}, \mathbf{y})$ and $V(\mathbf{x}, \mathbf{y})$ versus $\sigma$.

Also, Figs. 1 and 3 show distinct influences of the partial measures $M$ and $V$ in contributing to the global index $S$, depending on the nonlinear operation. In particular, an approximation $S \approx C V$ inspired from [19] is not necessarily accurate at all noise levels $\sigma$ when $M$ significantly varies with $\sigma$.

\section{CONCLUSION}

The present study is the first demonstration of the capability of the SSIM index of [1], [2] to register an effect of stochastic resonance or improvement by noise in nonlinear image transmission. A possible extension now is to apply SSIM on blocks or patches or across scales [1], [2], [20], [19], in order to complement with a more local or regional evaluation at relevant subscales to be identified, the global assessment of the stochastic resonance obtained here on the images.

Although still in an early stage, the SSIM index, from the recent results [1], [20], [2], [21], [22], [18], [23], [24], [19], is gradually emerging as a specially useful metric for image quality, providing a specific intermediate approach exhibiting richer capabilities for structural assessment compared to simpler traditional low-level-based indices, while avoiding the complexity of modeling the human visual system. The present results, by testing its behavior in a new task of image processing, reinforces this position of SSIM as a valuable quality metric. Especially, the assessment of stochastic resonance by SSIM does not copy the assessments by traditional low-level-based indices, confirming its specific capabilities as a quality metric. At the same time, the assessment by SSIM appears to better match the visual appreciation of image quality in the stochastic resonance experiments. These two features of SSIM (specificity of behavior and good match with visual perception) were generally preserved when varying the images and types of noise in our stochastic resonance experiments. The present results thus contribute to establish SSIM as an index for image quality with many useful potentialities of application. Also, the results complement the possible approaches for the on-going analyses of stochastic resonance or improvement by noise in image transmission, with a new metric enabling structural assessment of images and with relevance toward visual perception. 


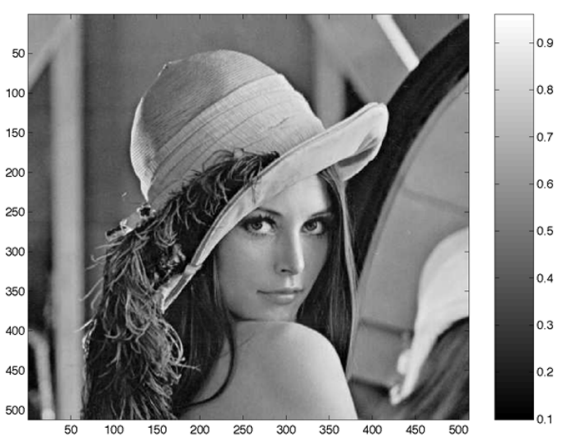

(a)

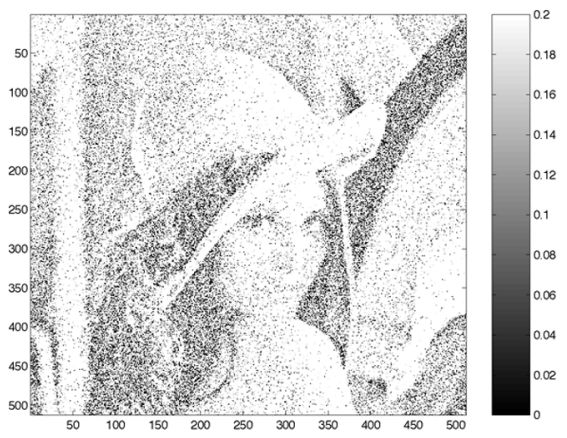

(d)

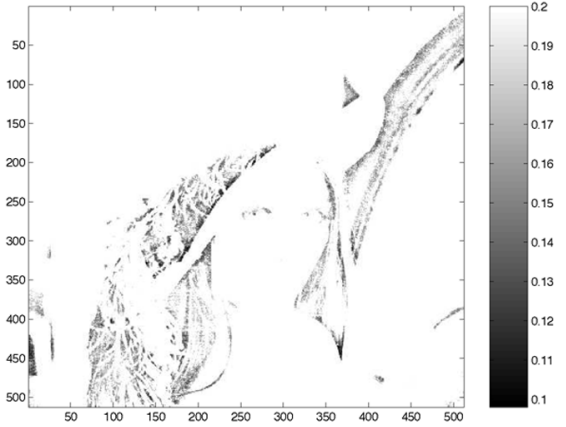

(b)

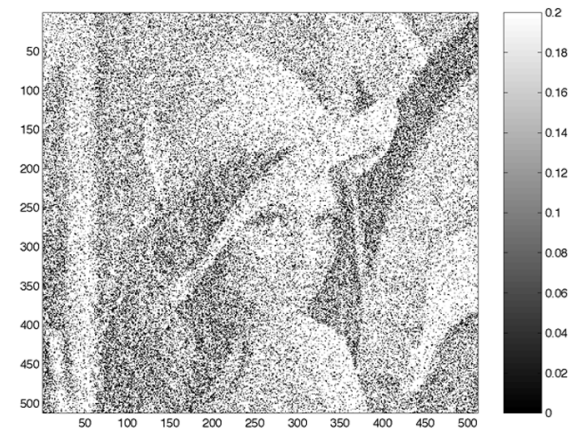

(e)

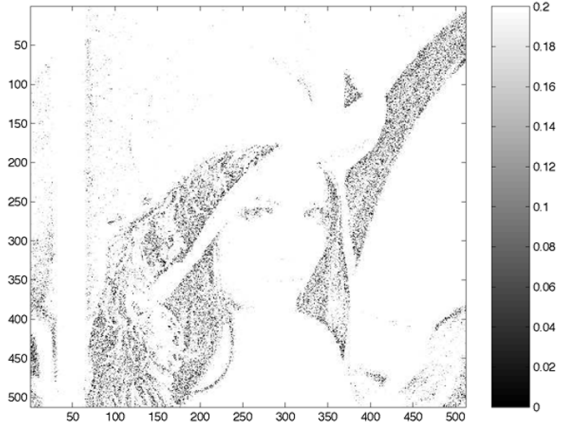

(c)

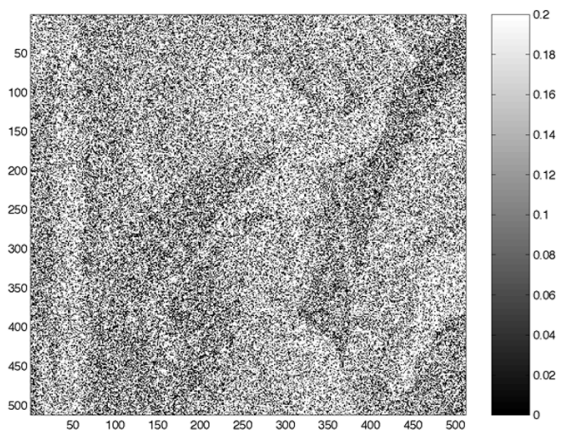

(f)

Fig. 4. (a) Gray-level input image $\mathbf{x}$. (b)-(f) gray-level image $\mathbf{y}$ at the output of the sensor of (6) with saturation level $\theta=0.2$, with the noise $\mathbf{n}$ zero-mean Gaussian of rms amplitude $\sigma$. (b) $\sigma=0$ maximizing the cross-correlation $C(\mathbf{x}, \mathbf{y})$ and minimizing the rms error $E(\mathbf{x}, \mathbf{y})$ in Fig. 3. (c) $\sigma=0.1$. (d) $\sigma=0.28$ maximizing the SSIM index $S(\mathbf{x}, \mathbf{y})$ in Fig. 3. (e) $\sigma=0.5$. (f) $\sigma=1$.

\section{REFERENCES}

[1] Z. Wang and A. C. Bovik, "A universal image quality index," IEEE Signal Process. Lett., vol. 9, pp. 81-84, 2002.

[2] Z. Wang, A. C. Bovik, H. R. Sheikh, and E. P. Simoncelli, "Image quality assessment: From error visibility to structural similarity," IEEE Trans. Image Process., vol. 13, pp. 600-612, 2004.

[3] B. Andò and S. Graziani, "Adding noise to improve measurement," IEEE Instrumen. Meas. Mag., vol. 4, no. 1, pp. 24-30, Mar. 2001.

[4] G. P. Harmer, B. R. Davis, and D. Abbott, "A review of stochastic resonance: Circuits and measurement," IEEE Trans. Instrum. Meas., vol. 51, pp. 299-309, 2002.

[5] F. Chapeau-Blondeau and D. Rousseau, "Noise improvements in stochastic resonance: From signal amplification to optimal detection," Fluct. Noise Lett., vol. 2, pp. L221-L233, 2002.

[6] E. Simonotto, M. Riani, C. Seife, M. Roberts, J. Twitty, and F. Moss, "Visual perception of stochastic resonance," Phys. Rev. Lett., vol. 78, pp. 1186-1189, 1997.

[7] F. Vaudelle, J. Gazengel, G. Rivoire, X. Godivier, and F. ChapeauBlondeau, "Stochastic resonance and noise-enhanced transmission of spatial signals in optics: The case of scattering," J. Opt. Soc. Amer. B, vol. 15, pp. 2674-2680, 1998.

[8] M. O. Hongler, Y. L. de Meneses, A. Beyeler, and J. Jacot, "The resonant retina: Exploiting vibration noise to optimally detect edges in an image," IEEE Trans. Pattern Anal. Machine Intell., vol. 25, pp. $1051-1062,2003$.

[9] A. Histace and D. Rousseau, "Constructive action of noise for impulsive noise removal in scalar images," Electron. Lett., vol. 42, pp. 393-395, 2006.

[10] S. Blanchard, D. Rousseau, D. Gindre, and F. Chapeau-Blondeau, "Constructive action of the speckle noise in a coherent imaging system," Opt. Lett., vol. 32, pp. 1983-1985, 2007.

[11] Y. Yang, Z. Jiang, B. Xu, and D. W. Repperger, "An investigation of two-dimensional parameter-induced stochastic resonance and applications in nonlinear image processing," J. Phys. A, vol. 42, pp. 145207,1-145207,9, 2009.

[12] T. Yang, "Adaptively optimizing stochastic resonance in visual system," Phys. Lett. A, vol. 245, pp. 79-86, 1998.
[13] M. Piana, M. Canfora, and M. Riani, "Role of noise in image processing by the human perceptive system," Phys. Rev. E, vol. 62, pp. 1104-1109, 2000.

[14] H. Sasaki, M. Todorokihara, T. Ishida, J. Miyachi, T. Kitamura, and $\mathrm{R}$. Aoki, "Effect of noise on the contrast detection threshold in visual perception," Neurosci. Lett., vol. 408, pp. 94-97, 2006.

[15] L. Gammaitoni, "Stochastic resonance and the dithering effect in threshold physical systems," Phys. Rev. E, vol. 52, pp. 4691-4698, 1995.

[16] F. Chapeau-Blondeau and X. Godivier, "Theory of stochastic resonance in signal transmission by static nonlinear systems," Phys. Rev. $E$, vol. 55, pp. 1478-1495, 1997.

[17] R. A. Wannamaker, S. P. Lipshitz, and J. Vanderkooy, "Stochastic resonance as dithering," Phys. Rev. E, vol. 61, pp. 233-236, 2000.

[18] Z. Wang, A. C. Bovik, and H. R. Sheikh, "Structural similarity based image quality assessment," in Digital Video Image Quality and Perceptual Coding, H. R. Wu and K. R. Rao, Eds. Boca Raton, FL: Taylor \& Francis, 2006, pp. 225-236, ch. 7

[19] D. M. Rouse and S. S. Hemami, "Understanding and simplifying the structural similarity metric," in Proc. 15th IEEE Int. Conf. Image Processing, San Diego, CA, Oct. 12-15, 2008, pp. 1188-1191.

[20] Z. Wang, E. P. Simoncelli, and A. C. Bovik, "Multiscale structural similarity for image quality assessment," in Proc. 37th IEEE Asilomar Conf. Signals, Systems and Computers, Pacific Grove, CA, Nov. 9-12, 2003, vol. 2, pp. 1398-1402.

[21] Z. Wang, L. Lu, and A. C. Bovik, "Video quality assessment based on structural distortion measurement," Signal Process.: Image Commun., vol. 19, pp. 121-132, 2004.

[22] D. Kalenova, D. Dochev, V. Bochko, P. Toivanen, and A. Kaarna, "A novel technique of spectral image quality assessment based on structural similarity measure," in Proc. 3rd Eur. Conf. Color in Graphics, Imaging and Vision, Leeds, UK, June 19-22, 2006, pp. 499-503.

[23] A. C. Brooks and T. N. Pappas, "Using structural similarity quality metrics to evaluate image compression techniques," in Proc. 32nd IEEE Int. Conf. Acoustics, Speech, and Signal Processing (ICASSP), Honolulu, HI, Apr. 15-20, 2007, vol. 1, pp. 873-876.

[24] J. H. Lee and T. Horiuchi, "Image quality assessment for color halftone images based on color structural similarity," IEICE Trans. Fund. Electron., Commun. Comput. Sci., vol. E91-A, pp. 1392-1399, 2008. 